\title{
Prophylaxis with amiodarone for postoperative atrial fibrillation: when and who?
}

\author{
Willemijn F. B. van der Does, Natasja M. S. de Groot \\ Department of Cardiology, Erasmus Medical Center Rotterdam, Rotterdam, The Netherlands \\ Correspondence to: Natasja M. S. de Groot. Unit Translational Electrophysiology, Department of Cardiology, Erasmus Medical Center, Dr. \\ Molewaterplein 40, 3015 GD Rotterdam, The Netherlands. Email: n.m.s.degroot@erasmusmc.nl. \\ Comment on: Mehaffey JH, Hawkins RB, Byler M, et al. Amiodarone Protocol Provides Cost-Effective Reduction in Postoperative Atrial Fibrillation. \\ Ann Thorac Surg 2018;105:1697-702.
}

Submitted Aug 17, 2018. Accepted for publication Sep 11, 2018.

doi: $10.21037 /$ jtd.2018.10.14

View this article at: http://dx.doi.org/10.21037/jtd.2018.10.14

Post-operative atrial fibrillation (PoAF) is generally considered as a benign, self-limiting complication of cardiac surgery. However, it is associated with complications such as stroke, prolonged intensive care unit (ICU) stay and need for dialysis, leading to longer hospitalization and higher costs $(1,2)$. Even in-hospital and long-term mortality are higher in patients who develop PoAF $(2,3)$, making it clear that PoAF is less innocent than assumed.

Therefore, pathophysiology and prevention of PoAF have been topics of extensive research. In this issue of Fournal of thoracic disease (FTD), Mehaffey et al. (4) give a detailed report of their retrospective evaluation of costeffectiveness of a prophylactic amiodarone protocol implemented to reduce the incidence of PoAF. They found a significantly reduced rate of PoAF and $\$ 458$ cost saving per patient with the implemented protocol. They did not observe a significant increase in risk-adjusted adverse events related to amiodarone.

The findings of cost saving by prevention of PoAF with amiodarone are in accordance with prior studies (5-7), although this is the first cost-effectiveness analysis of a protocol-driven approach. The authors are to be complemented for their clear example of assessing costeffectiveness of an implemented quality improvement protocol. Important to mention is the high percentage of patients who were treated according to the protocol. As also remarked by the authors, this can be attributed to the protocol starting immediately postoperatively. But correct timing of amiodarone prophylaxis is a matter to consider.

\section{Timing and dosage of amiodarone}

Research into the pathophysiology of PoAF has identified chronic and acute factors at play (8). As soon as the surgeon cuts in the patient's tissue the acute factors, related to the surgical intervention, become relevant. Therefore, it would be rational to presume that appropriate tissue levels of amiodarone at this moment in time are a prerequisite for maximal prevention of PoAF. However, two meta-analyses focusing on the timing of amiodarone administration did not find a difference between a post-operative or preoperative start of amiodarone in coronary artery bypass graft $(\mathrm{CABG})$ patients $(9,10)$. A possible explanation for this is that onset of PoAF is typically $2-3$ days after cardiac surgery (2). This suggests that pro-arrhythmic mechanisms related to the surgery, such as inflammation, oxidative stress and sympathetic activation, reach their maximum impact at this time. Therefore, starting amiodarone immediately post-operatively, allows enough time to reach the adequate tissue levels.

Dosage and route of administration of amiodarone were discussed in meta-analyses by Buckley et al. and Chatterjee et al. respectively. Total prophylactic amiodarone dosage above $3,000 \mathrm{mg}$ was found to be effective by Buckley et al. (9). Chatterjee et al. did not find a difference between regimens with only oral amiodarone or a combination of oral and intravenous amiodarone (10). By these standards, the regimen used by Mehaffey et al. is appropriate.

When addressing the timing of initiation of the protocol, 
differences in clinical endpoints are of importance. In the current study, it was reported that length of hospital stay and major morbidity in the two groups were not significantly different. Yet, clinical endpoints for interventions reducing PoAF incidence were analyzed in various meta-analyses showing positive effects for amiodarone prophylaxis on incidence of stroke, occurrence of ventricular tachycardia and length of hospital stay $(7,11,12)$. However, these metaanalyses did not divide the studies in pre or post-operative start of amiodarone prophylaxis. Prior studies with a preoperative as well as a post-operative start of amiodarone protocol showed a significant reduction in length of stay (13-15). Yet, a lower incidence of post-operative stroke was only found in regimens starting pre-operatively (16). So far, there are no reports on the different timing of the prophylaxis in relation to these hard-clinical endpoints, though they are needed to further validate the use of the various protocols.

\section{Substrate of PoAF}

Some patients have a higher risk of developing PoAF than others and various risk factors have been determined (2). Distinguishing patients at risk could improve the benefit of implemented protocols. One retrospective observational analysis by Barnes et al. used a previously validated risk score for PoAF to investigate cost-effectiveness of amiodarone prophylaxis. Patients were stratified into 3 risk groups according to risk-increasing variables such as advancing age, a history of COPD or undergoing heart valve surgery. Also, riskdecreasing variables such as postoperative beta-blocker use were taken into account. They found that the greatest benefits were seen in patients at high risk for developing PoAF, and in that group prophylaxis was most cost-effective (17).

As mentioned above, acute as well as chronic factors contribute to development of PoAF. Determining the presence of these chronic factors or, as you could say, determining the presence of an arrhythmogenic substrate for PoAF in the atria could more accurately pinpoint patients at risk. A considerable amount of electrophysiological mapping studies is aimed at identifying the electrophysiological substrate of atrial fibrillation, although studies focusing on the substrate of specifically PoAF are rare. Sakamoto et al. obtained epicardial recordings of the right atrium in sinus rhythm from 52 patients. These patients underwent cardiac surgery for valvular disease, ischemic heart disease or a congenital heart disease. They found that a non-uniform activation pattern was a significant independent predictor for PoAF (18). Teuwen et al performed high-resolution epicardial mapping of Bachmann's bundle (BB) during sinus rhythm in 185 patients during coronary artery bypass surgery and found that a high amount of conduction block was associated with PoAF (19).

In discovering what (electrophysiological) features of the atria define the substrate for PoAF, our understanding of the pathophysiology will grow and we can more accurately predict which patient will develop PoAF and which patient will not.

\section{Acknowledgements}

None.

\section{Footnote}

Conflicts of Interest: The authors have no conflicts of interest to declare.

\section{References}

1. Lahtinen J, Biancari F, Salmela E, et al. Postoperative atrial fibrillation is a major cause of stroke after onpump coronary artery bypass surgery. Ann Thorac Surg 2004;77:1241-4.

2. Mathew JP, Fontes ML, Tudor IC, et al. A multicenter risk index for atrial fibrillation after cardiac surgery. JAMA 2004;291:1720-9.

3. El-Chami MF, Kilgo P, Thourani V, et al. New-onset atrial fibrillation predicts long-term mortality after coronary artery bypass graft. J Am Coll Cardiol 2010;55:1370-6.

4. Mehaffey JH, Hawkins RB, Byler M, et al. Amiodarone Protocol Provides Cost-Effective Reduction in Postoperative Atrial Fibrillation. Ann Thorac Surg 2018;105:1697-702.

5. Gillespie EL, White CM, Kluger J, et al. Costeffectiveness of amiodarone for prophylaxis of atrial fibrillation after cardiothoracic surgery. Pharmacotherapy 2006;26:499-504.

6. Zebis LR, Christensen TD, Kristiansen IS, et al. Amiodarone cost effectiveness in preventing atrial fibrillation after coronary artery bypass graft surgery. Ann Thorac Surg 2008;85:28-32.

7. Arsenault KA, Yusuf AM, Crystal E, et al. Interventions for preventing post-operative atrial fibrillation in patients undergoing heart surgery. Cochrane Database Syst Rev 2013:CD003611. 
8. Maesen B, Nijs J, Maessen J, et al. Post-operative atrial fibrillation: a maze of mechanisms. Europace 2012;14:159-74.

9. Buckley MS, Nolan PE Jr, Slack MK, et al. Amiodarone prophylaxis for atrial fibrillation after cardiac surgery: meta-analysis of dose response and timing of initiation. Pharmacotherapy 2007;27:360-8.

10. Chatterjee S, Sardar P, Mukherjee D, et al. Timing and route of amiodarone for prevention of postoperative atrial fibrillation after cardiac surgery: a network regression metaanalysis. Pacing Clin Electrophysiol 2013;36:1017-23.

11. Bagshaw SM, Galbraith PD, Mitchell LB, et al. Prophylactic amiodarone for prevention of atrial fibrillation after cardiac surgery: a meta-analysis. Ann Thorac Surg 2006;82:1927-37.

12. Burgess DC, Kilborn MJ, Keech AC. Interventions for prevention of post-operative atrial fibrillation and its complications after cardiac surgery: a meta-analysis. Eur Heart J 2006;27:2846-57.

13. Roshanali F, Mandegar MH, Yousefnia MA, et al. Prevention of atrial fibrillation after coronary artery bypass grafting via atrial electromechanical interval and use of amiodarone prophylaxis. Interact Cardiovasc Thorac Surg
2009;8:421-5.

14. Yagdi T, Nalbantgil S, Ayik F, et al. Amiodarone reduces the incidence of atrial fibrillation after coronary artery bypass grafting. J Thorac Cardiovasc Surg 2003;125:1420-5.

15. Daoud EG, Strickberger SA, Man KC, et al. Preoperative amiodarone as prophylaxis against atrial fibrillation after heart surgery. N Engl J Med 1997;337:1785-91.

16. Giri S, White CM, Dunn AB, et al. Oral amiodarone for prevention of atrial fibrillation after open heart surgery, the Atrial Fibrillation Suppression Trial (AFIST): a randomised placebo-controlled trial. Lancet 2001;357:830-6.

17. Barnes BJ, Kirkland EA, Howard PA, et al. Risk-stratified evaluation of amiodarone to prevent atrial fibrillation after cardiac surgery. Ann Thorac Surg 2006;82:1332-7.

18. Sakamoto S, Yamauchi S, Yamashita H, et al. Intraoperative mapping of the right atrial free wall during sinus rhythm: variety of activation patterns and incidence of postoperative atrial fibrillation. Eur J Cardiothorac Surg 2006;30:132-9.

19. Teuwen CP, Yaksh A, Lanters EA, et al. Relevance of Conduction Disorders in Bachmann's Bundle During Sinus Rhythm in Humans. Circ Arrhythm Electrophysiol 2016;9:e003972.
Cite this article as: van der Does WF, de Groot NM. Prophylaxis with amiodarone for postoperative atrial fibrillation: when and who? J Thorac Dis 2018;10(Suppl 33):S3831-S3833. doi: $10.21037 /$ jtd.2018.10.14 